\title{
ON NONLOCAL BOUNDARY VALUE PROBLEM FOR THE EQUATION OF MOTION OF A HOMOGENEOUS ELASTIC BEAM WITH PINNED-PINNED ENDS
}

In the current paper, in the domain $D=\{(t, x): t \in(0, T), x \in(0, L)\}$ we investigate the boundary value problem for the equation of motion of a homogeneous elastic beam

$$
u_{t t}(t, x)+a^{2} u_{x x x x}(t, x)+b u_{x x}(t, x)+c u(t, x)=0,
$$

where $a, b, c \in \mathbb{R}, b^{2}<4 a^{2} c$, with nonlocal two-point conditions

$$
u(0, x)-u(T, x)=\varphi(x), \quad u_{t}(0, x)-u_{t}(T, x)=\psi(x)
$$

and local boundary conditions $u(t, 0)=u(t, L)=u_{x x}(t, 0)=u_{x x}(t, L)=0$. Solvability of this problem is connected with the problem of small denominators, whose estimation from below is based on the application of the metric approach. For almost all (with respect to Lebesgue measure) parameters of the problem, we establish conditions for the solvability of the problem in the Sobolev spaces. In particular, if $\varphi \in \mathbf{H}_{q+\rho+2}$ and $\psi \in \mathbf{H}_{q+\rho}$, where $\rho>2$, then for almost all (with respect to Lebesgue measure in $\mathbb{R})$ numbers $a$ there exists a unique solution $u \in \mathbf{C}^{2}\left([0, T] ; \mathbf{H}_{q}\right)$ of the problem.

Key words and phrases: nonlocal boundary value problem, homogeneous beam, small denominator, Lebesque measure, metric approach.

\footnotetext{
${ }^{1}$ Vasyl Stefanyk Precarpathian National University, 57 Shevchenka str., 76018, Ivano-Frankivsk, Ukraine

2 Pidstryhach Institute for Applied Problems of Mechanics and Mathematics, 3b Naukova str., 79060, Lviv, Ukraine

E-mail: tarasgoy@yahoo.com(Goy T.), negrychmariya@gmail.com(Negrych M.), s-i@ukr.net (Savka I.)
}

\section{INTRODUCTION}

Among nonclassical boundary value problems for partial differential equations, the problems with nonlocal conditions occupy an important place, including those that connect the values of required solutions and its derivatives in at least two boundary or interior points of the domain considered. The periodicity conditions are the simplest of these conditions.

A general definition of nonlocal conditions and their classification were introduced by Nakhushev [11].

Nonlocal problems for partial differential equations are usually ill-posed in Hadamard's sense, and their solvability (in case of a bounded domain) is connected with the problem of small denominators and is unstable with respect to small variations both in the coefficients of the problem and the parameters of the domain.

Azizbayov and Mehraliyev [1,2] studied the nonlocal problems for the nonlinear equation of motion of a homogeneous elastic beam in a rectangle. By using the contracting mappings principle, the authors proved the existence and uniqueness of the solution in case $|\delta| \neq 1$, 
where $\delta$ is the parameter of nonlocal conditions. Sabitov [13] investigated the Cauchy problem for the equation of the beam's motion with clamped ends. In particular, theorems of the existence and uniqueness of the solution in the classes of generalized and regular functions are proved.

The present paper is based on ideas close to those used in $[6,16]$. The conditions of solvability of nonlocal problem in the time variable $t$ for fourth order hyperbolic equation in a rectangle are established. The metric approach is used to find the lower bounds of small denominators appearing in the solution of the problem.

For some classes of equations and systems of equations, non-regular class of problems with nonlocal conditions with respect to a select variable $t$ were investigated among others in $[5-9,12,14,15,17-19]$.

\section{PROBLEM STATEMENT}

In the domain $D=\{(t, x): t \in(0, T), x \in(0, L)\}$ we consider the problem for the equation of wave motion of an elastic beam:

$$
P(u) \equiv u_{t t}(t, x)+a^{2} u_{x x x x}(t, x)+b u_{x x}(t, x)+c u(t, x)=0, \quad(t, x) \in D,
$$

with local conditions of pinned-pinned ends

$$
u(t, 0)=u(t, L)=u_{x x}(t, 0)=u_{x x}(t, L)=0, \quad 0 \leq t \leq T,
$$

and two-point nonlocal boundary conditions

$$
\begin{aligned}
& Q_{1}(u) \equiv u(0, x)-u(T, x)=\varphi(x), \quad 0 \leq x \leq L, \\
& Q_{2}(u) \equiv u_{t}(0, x)-u_{t}(T, x)=\psi(x), \quad 0 \leq x \leq L,
\end{aligned}
$$

where $T>0, L>0, a, b, c \in \mathbb{R}$, moreover $b^{2}<4 a^{2} c, \varphi$ and $\psi$ are given functions.

Note that many problems about vibrations of beams and platens in structural mechanics lead to higher order differential equations than equation of rod $[1,2,10,13]$. In particular, the equation (1) of fourth order can model the dynamic response of homogeneous beam on an elastic foundation with axial loading [3].

Below, we use the following functional spaces:

$\mathbf{H}_{q}=\mathbf{H}_{q}[0, L]$, where $q \in \mathbb{R}$, is the Sobolev space of all trigonometric series $\varphi(x)=$ $\sum_{k \in \mathbb{N}} \varphi_{k} \sin \frac{k \pi x}{L}$ with the norm $\|\varphi\|_{\mathbf{H}_{q}}=\left(\sum_{k \in \mathbb{N}} k^{2 q}\left|\varphi_{k}\right|^{2}\right)^{1 / 2}$;

$\mathbf{C}^{n}\left([0, T] ; \mathbf{H}_{q}\right), n \in \mathbb{Z}_{+}$, is the space of all series

$$
u(t, x)=\sum_{k \in \mathbb{N}} u_{k}(t) \sin \frac{k \pi x}{L}
$$

where $u_{k} \in \mathbf{C}^{n}[0, T], k \in \mathbb{N}$, with the norm

$$
\|u\|_{\mathbf{C}^{n}\left([0, T] ; \mathbf{H}_{q}\right)}^{2}=\sum_{j=0}^{n} \max _{t \in[0, T]}\left\|\sum_{k \in \mathbb{N}} u_{k}^{(j)}(t) \sin \frac{k \pi x}{L}\right\|_{\mathbf{H}_{q}}^{2} .
$$

A function $u=u(t, x)$ from $\mathbf{C}^{2}\left([0, T] ; \mathbf{H}_{q}\right)$ is called a solution of the problem (1)-(3), if it satisfies conditions

$$
\|P(u)\|_{\mathbf{C}^{2}\left([0, T] ; \mathbf{H}_{q}\right)}=0, \quad\left\|Q_{1}(u)-\varphi\right\|_{\mathbf{H}_{q}}=0, \quad\left\|Q_{2}(u)-\psi\right\|_{\mathbf{H}_{q-2}}=0 .
$$




\section{THE CONDITIONS OF UNIQUENESS OF THE SOLUTION}

We seek a solution of the problem (1)-(3) in the form of a series

$$
u(t, x)=\sum_{k \in \mathbb{N}} u_{k}(t) \sin \lambda_{k} x,
$$

where $\lambda_{k}=\frac{\pi k}{L}$. Each function $u_{k}(t), k \in \mathbb{N}$, in (4) is a solution of the problem

$$
\begin{gathered}
u_{k}^{\prime \prime}(t)+\left(a^{2} \lambda_{k}^{4}-b \lambda_{k}^{2}+c\right) u_{k}(t)=0, \\
u_{k}(0)-u_{k}(T)=\varphi_{k}, \quad u_{k}^{\prime}(0)-u_{k}^{\prime}(T)=\psi_{k},
\end{gathered}
$$

where $\varphi_{k}$ and $\psi_{k}$ are the Fourier coefficients of the functions $\varphi$ and $\psi$ (in the system $\left\{\sin \lambda_{k} x\right\}$, $k \in \mathbb{N})$.

The characteristic determinant $\Delta_{k}$ of the problem (5), (6) is defined by

$$
\Delta_{k}=2 \beta_{k}\left(1-\cos \beta_{k} T\right) \text {, }
$$

where $\beta_{k}=\sqrt{a^{2} \lambda_{k}^{4}-b \lambda_{k}^{2}+c}, k \in \mathbb{N}$.

Denote $\mathbb{N}_{0}=\left\{k \in \mathbb{N}: \Delta_{k}=0\right\}$. If $\varphi_{k}=0$ and $\psi_{k}=0$ for $k \in \mathbb{N}_{0}$, then the solution of the problem (5), (6) is not unique and it is defined by

$$
u_{k}(t)=C_{1 k} \cos \beta_{k} t+C_{2 k} \sin \beta_{k} t,
$$

where $C_{1 k}, C_{2 k}$ are arbitrary constants, $k \in \mathbb{N}_{0}$.

If $k \in \mathbb{N} \backslash \mathbb{N}_{0}$, then $C_{1 k}, C_{2 k}$ are solutions of the homogeneous systems of linear equations

$$
\left\{\begin{array}{l}
C_{1 k}\left(1-\cos \beta_{k} T\right)-C_{2 k} \sin \beta_{k} T=0, \\
C_{1 k} \beta_{k} \sin \beta_{k} T+C_{2 k}\left(1-\cos \beta_{k} T\right)=0
\end{array}\right.
$$

and functions $u_{k}(t)$ have the form

$$
u_{k}(t)=\frac{\beta_{k}\left(\cos \beta_{k} t-\cos \beta_{k}(t-T)\right) \varphi_{k}+\left(\sin \beta_{k} t+\sin \beta_{k}(t+T)\right) \psi_{k}}{2 \beta_{k}\left(1-\cos \beta_{k} T\right)} .
$$

If $\beta_{k} T \neq 2 \pi m$ for all $(k, m) \in \mathbb{N}^{2}$, then set $\mathbb{N}_{0}$ is empty and functions $u_{k}(t)$ are defined by the formula (8).

Theorem 1. For uniqueness of the solution $u \in \mathbf{C}^{2}\left([0, T] ; \mathbf{H}_{q}\right)$ of the problem (1)-(3) it is necessary and sufficient that the following condition should be satisfied:

$$
\left(\text { for all }(k, m) \in \mathbb{N}^{2}\right) \quad \beta_{k} T \neq 2 \pi m \text {. }
$$

The proof follows from uniqueness of Fourier expansion of the functions with respect to the system $\left\{\sin \lambda_{k} x\right\}, k \in \mathbb{N}$.

Thus, if $\varphi_{k}=\psi_{k}=0$ for all $k \in \mathbb{N}_{0}$ then from (4), (7), (8) follows that the problem (1)-(3) has the formal solution defined by

$$
\begin{aligned}
u(t, x) & =\sum_{k \in \mathbb{N}_{0}}\left(C_{1 k} \cos \beta_{k} t+C_{2 k} \sin \beta_{k} t\right) \sin \lambda_{k} x \\
& +\sum_{k \in \mathbb{N} \backslash \mathbb{N}_{0}} \frac{\beta_{k}\left(\cos \beta_{k} t-\cos \beta_{k}(t-T)\right) \varphi_{k}+\left(\sin \beta_{k} t+\sin \beta_{k}(t+T)\right) \psi_{k}}{2 \beta_{k}\left(1-\cos \beta_{k} T\right)} \sin \lambda_{k} x .
\end{aligned}
$$

If conditions (9) are satisfied then the set $\mathbb{N}_{0}$ is empty and formal solution of the problem (1)-(3) is unique and is given as follows

$$
u(t, x)=\sum_{k \in \mathbb{N}} \frac{\beta_{k}\left(\cos \beta_{k} t-\cos \beta_{k}(t-T)\right) \varphi_{k}+\left(\sin \beta_{k} t+\sin \beta_{k}(t+T)\right) \psi_{k}}{2 \beta_{k}\left(1-\cos \beta_{k} T\right)} \sin \lambda_{k} x .
$$




\section{THE CONDITIONS OF EXISTENCE OF THE SOLUTION}

In what follows, we assume that conditions (9) are satisfied. Then there exists a unique solution of the problem (1)-(3), which admits a representation (11).

The existence of the solution $u \in \mathbf{C}^{2}\left([0, T] ; \mathbf{H}_{q}\right)$ of the problem (1)-(3) is associated with the problem of small denominators, because the terms of the sequence $\left\{1-\cos \beta_{k} T\right\}_{k \in \mathbb{N}}$ in the denominator of the formula (11), being different from zero, can rapidly approach zero if $k \rightarrow+\infty$. This leads to a divergence of series (11) in the space $\mathbf{C}^{2}\left([0, T] ; \mathbf{H}_{q}\right)$ and therefore we get unsolvable problem in preset scale.

If we can estimate below small denominators $1-\cos \beta_{k} T$ with certain or exponential behavior with respect to $k$, then the relevant restrictions on the functions $\varphi$ and $\psi$ we can obtain the correct solvability of the problem.

Lemma 1. For all $k \in \mathbb{N}$, the following estimates hold

$$
C_{1} k^{2} \leq \beta_{k} \leq C_{2} k^{2}
$$

where $C_{1}, C_{2}$ are constants independent of $k$.

Proof. Let $r_{1}, r_{2}$ are complex roots of the equation $a^{2} r^{2}-b r+c=0$. Then we get

$$
\begin{aligned}
\beta_{k}^{2} & =a^{2}\left|\lambda_{k}^{2}-r_{1}\right|\left|\lambda_{k}^{2}-r_{2}\right| \geq a^{2}\left|\lambda_{k}^{2}-\right| r_{1}||\left|\lambda_{k}^{2}-\right| r_{2}|| \\
& \geq a^{2}\left|\frac{1}{2} \lambda_{k}^{2}+\frac{1}{2} \lambda_{k}^{2}-\right| r_{1}||\left|\frac{1}{2} \lambda_{k}^{2}+\frac{1}{2} \lambda_{k}^{2}-\right| r_{2}|| \geq \frac{a^{2}}{4} \lambda_{k}^{4}=C_{3} k^{4}
\end{aligned}
$$

for all $k \geq K_{1}$, where $C_{3}=(a / 2)^{2}(\pi / L)^{4}$ and $K_{1}=\pi^{-1} \sqrt{2 L \max \left\{\left|r_{1}\right|,\left|r_{2}\right|\right.}$.

If $k<K_{1}$, then $\beta_{k}^{2} \geq C_{4} k^{4}$, where $C_{4}=\min _{k<K_{1}}\left\{\beta_{k}^{2} k^{-4}\right\}$. Thus, $\beta_{k} \geq C_{1} k^{2}$, for all $k \in \mathbb{N}$, where $C_{1}=\min \left\{\sqrt{C_{3}}, \sqrt{C_{4}}\right\}$.

Similarly, we obtain the estimate from above

$$
\beta_{k}^{2} \leq a^{2} \lambda_{k}^{4}+|b| \lambda_{k}^{4}+c \lambda_{k}^{4} \leq C_{5} k^{4}
$$

where $C_{5}=\left(a^{2}+|b|+c\right)\left(\frac{\pi}{L}\right)^{4}$. Therefore, $\beta_{k} \leq C_{2} k^{2}$, where $C_{2}=\sqrt{C_{5}}$.

Using Lemma 1, we prove existence of the solution of the problem (1)-(3).

Theorem 2. Let conditions (9) are satisfied and there exist numbers $C_{6}>0$ and $\gamma \in \mathbb{R}$ such that inequality

$$
\left|1-\cos \beta_{k} T\right| \geq C_{6} k^{-\gamma}
$$

is fulfilled for all (except finitely many numbers) $k \in \mathbb{N}$. If $\varphi \in \mathbf{H}_{q+\gamma+4}$ and $\psi \in \mathbf{H}_{q+\gamma+2 \text {, }}$ then exists a unique solution $u \in \mathbf{C}^{2}\left([0, T] ; \mathbf{H}_{q}\right)$ of the problem (1)-(3), which continuously depends on functions $\varphi$ and $\psi$, i.e.,

$$
\|u\|_{\mathbf{C}^{n}\left([0, T] ; \mathbf{H}_{q}\right)}^{2} \leq C_{7}\left(\|\varphi\|_{\left.\mathbf{H}_{q+\gamma+4}\right)}^{2}+\|\psi\|_{\left.\mathbf{H}_{q+\gamma+2}\right)}^{2}\right),
$$

where $C_{7}$ is a constant independent of $k$. 
Proof. From (8), (12), (13), we obtain the estimates

$$
\left|u_{k}^{(j)}(t)\right| \leq \frac{C_{2} C_{2}^{j} k^{2 j+2}\left|\varphi_{k}\right|+C_{2}^{j} k^{2 j}\left|\psi_{k}\right|}{C_{1} C_{6} k^{2-\gamma}} \leq C_{8} k^{2 j+\gamma-2}\left(k^{2}\left|\varphi_{k}\right|+\left|\psi_{k}\right|\right),
$$

for $j \in\{0,1,2\}$, where $C_{8}=\frac{\max \left\{1, C_{2}^{3}\right\}}{C_{1} C_{6}}$.

In view of the above estimate, we obtain the estimate for norm of the solution of the problem (1)-(3):

$$
\begin{aligned}
\|u\|_{\mathbf{C}^{2}\left([0, T] ; \mathbf{H}_{q}\right)}^{2} & =\sum_{j=0}^{2} \max _{t \in[0, T]}\left\|\sum_{k \in \mathbb{N}} u_{k}^{(j)}(t) \sin \lambda_{k} x\right\|_{\mathbf{H}_{q}}^{2} \\
& \leq \sum_{j=0}^{2} \sum_{k \in \mathbb{N}} k^{2 q} C_{8}^{2} k^{2(2 j+\gamma-2)}\left(k^{2}\left|\varphi_{k}\right|+\left|\psi_{k}\right|\right)^{2} \\
& =2 C_{8}^{2} \sum_{j=0}^{2} \sum_{k \in \mathbb{N}} k^{2(2 j+q+\gamma-2)}\left(k^{4}\left|\varphi_{k}\right|^{2}+\left|\psi_{k}\right|^{2}\right) \\
& \leq 6 C_{8}^{2} \sum_{k \in \mathbb{N}} k^{2(q+\gamma+2)}\left(k^{4}\left|\varphi_{k}\right|^{2}+\left|\psi_{k}\right|^{2}\right) \\
& =6 C_{8}^{2} \sum_{k \in \mathbb{N}}\left(k^{2(q+\gamma+4)}\left|\varphi_{k}\right|^{2}+k^{2(q+\gamma+2)}\left|\psi_{k}\right|^{2}\right)=C_{7}\left(\|\varphi\|_{\mathbf{H}_{q+\gamma+4}}^{2}+\|\psi\|_{\mathbf{H}_{q+\gamma+2}}^{2}\right) .
\end{aligned}
$$

This completes the proof.

\section{THE METRIC ESTIMATES OF THE SMALL DENOMINATORS}

In Theorem 2, the condition (13) is imposed axiomatically. Now we study the conditions of validity of the inequalities (13).

To this end, we use the following assertion. Let meas $A$ is the Lebesgue measure of a measurable set $A$.

Lemma 2 (Borel-Cantelli [4]). Let $\left\{A_{k}\right\}_{k \in \mathbb{N}}$ be a countable collection of measurable sets such that $\sum_{k \in \mathbb{N}}$ meas $A_{k}<\infty$. Then the set of points that belongs to an infinite number of the sets $A_{k}$ has zero Lebesgue measure.

Lemma 3 ([16]). Let $f \in C^{1}\left[t_{1}, t_{2}\right]$. If $\left|f^{\prime}(t)\right| \geq \delta>0$ for all $t \in\left[t_{1}, t_{2}\right]$, then

$$
\operatorname{meas}\left\{t \in\left[t_{1}, t_{2}\right]:|f(t)|<\varepsilon\right\} \leq \frac{2 \varepsilon}{\delta}
$$

where $\varepsilon>0$.

\subsection{The estimates in terms of coefficients $a, b, c$.}

Let $a \in\left[a_{1}, a_{2}\right], 0<a_{1}<a_{2}$, and $b, c$ are arbitrary fixed numbers in (1).

Theorem 3. For almost all (with respect to Lebesgue measure in $\mathbb{R}$ ) numbers $a \in\left[a_{1}, a_{2}\right]$ inequality

$$
\left|1-\cos \beta_{k} T\right| \geq 2 k^{-\gamma}
$$

is fulfilled for all (except finitely many numbers) $k \in \mathbb{N}$ and $\gamma>0$. 
Proof. Since $1-\cos \beta_{k} T=2 \sin ^{2} \frac{\beta_{k} T}{2}$, it follows that establishment of estimate (14) is reduced to establishment of estimate underneath for sequence

$$
f_{k}(a)=\sin \frac{\beta_{k} T}{2}, \quad k \in \mathbb{N},
$$

where $\beta_{k}=\beta_{k}(a)=\sqrt{a^{2} \lambda_{k}^{4}-b \lambda_{k}^{2}+c}, a \in\left[a_{1}, a_{2}\right]$.

For every fixed $k \in \mathbb{N}$ we introduce the set $A_{k}=\left\{a \in\left[a_{1}, a_{2}\right]:\left|f_{k}(a)\right|<k^{-\theta}, \theta>0\right\}$. Let $A$ denote the set of numbers which are infinite number of sets $A_{k}, k \in \mathbb{N}$. We find the estimate for measure of set $A_{k}$ : for $k>1$,

$$
\left|f_{k}^{\prime}(a)\right|=\frac{a T \lambda_{k}^{4}}{2 \beta_{k}} \sqrt{1-\sin ^{2} \frac{\beta_{k} T}{2}} \geq \frac{a_{1} T \lambda_{k}^{4}}{2 \beta_{k}} \sqrt{1-k^{-2 \theta}} \geq \frac{a_{1} T\left(\frac{k \pi}{L}\right)^{4}}{2 C_{2} k^{2}} \sqrt{1-2^{-2 \theta}}=C_{9} k^{2},
$$

where $C_{9}=\frac{a_{1} T}{2 C_{2}}\left(\frac{\pi}{L}\right)^{4} \sqrt{1-2^{-2 \theta}}>0$. Hence, for every $k>1,\left|f_{k}^{\prime}(a)\right| \geq C_{9} k^{2}$. Then for every $k>1$, by Lemma 3, we obtain the estimate for measure of set $A_{k}$ as follows

$$
\text { meas } A_{k} \leq \frac{2 k^{-\theta}}{C_{9} k^{2}}=\frac{2}{C_{9} k^{2+\theta}} .
$$

For fixed $\theta>0$, series $\sum_{k>1}$ meas $A_{k}$ is majorized by the convergent series $\frac{2}{C_{9}} \sum_{k>1} \frac{1}{k^{2+\theta}}$. Then from Lemma 2 we conclude that Lebesgue measure of the set of points $a \in\left[a_{1}, a_{2}\right]$, which contained the infinite numbers of sets $A_{k}$, is equal to zero.

Thus, meas $A=0$. So, for $a \in\left[a_{1}, a_{2}\right] \backslash A$ there exists number $k=K(a)$, that the estimate $\left|f_{k}(a)\right| \geq k^{-\theta}$ is valid for all $k \geq K(a)$ and $\theta>0$. Then $\left|1-\cos \beta_{k} T\right| \geq 2 k^{-\gamma}$, where $\gamma=2 \theta$.

Theorem 4. For almost all (with respect to Lebesgue measure in $\mathbb{R}$ ) numbers $a \in\left[a_{1}, a_{2}\right]$ conditions (9) are fulfilled.

Proof. Let us consider the set $B_{k}=\left\{a \in\left[a_{1}, a_{2}\right]: \cos \beta_{k}(a) T=1\right\}$, for fixed $k$. Since $\beta_{k}(a)=$ $\sqrt{a^{2} \lambda_{k}^{4}-b \lambda_{k}^{2}+c}$, we have

$$
a^{2} \lambda_{k}^{4}-b \lambda_{k}^{2}+c=\left(\frac{2 \pi m}{T}\right)^{2}, \quad m \in \mathbb{N}
$$

Since equation (1) is hyperbolic, we conclude that equation (15) can not have more than two real roots relatively variable $a$ for fixed $k$.

Let $B_{k}^{m}$ be the set of roots of the equation (15). Obviously, that

$$
B_{k}=\bigcup_{m \in \mathbb{N}} B_{k}^{m}, \quad \text { meas } B_{k} \leq \sum_{m \in \mathbb{N}} \text { meas } B_{k}^{m} .
$$

Since meas $B_{k}^{m}=0$ we conclude that $B_{k}=0$. We introduce the set

$$
B=\left\{a \in\left[a_{1}, a_{2}\right]: \prod_{k \in \mathbb{N}}\left(1-\cos \beta_{k}(a) T\right)=0\right\} .
$$

As well as $B=\bigcup_{k \in \mathbb{N}} B_{k}$, it follows that meas $B=0$.

Therefore, $\beta_{k} T \neq 2 \pi m, m \in \mathbb{N}$, for almost all $a \in\left[a_{1}, a_{2}\right]$. 
Corollary 1. For almost all (with respect to Lebesgue measure in $\mathbb{R}$ ) numbers $a \in\left[a_{1}, a_{2}\right]$, inequality

$$
\left|1-\cos \beta_{k} T\right| \geq C_{10} k^{-\gamma}, \quad C_{10}>0,
$$

holds for $\gamma>0$ and $k \in \mathbb{N}$.

Proof. If $a \in\left[a_{1}, a_{2}\right] \backslash(A \cup B)$ then from Theorems 3 and 4 we obtain

$$
\begin{aligned}
\left|1-\cos \beta_{k} T\right| & \geq 2 k^{-\gamma}, \quad k \geq K(a), \\
\left|1-\cos \beta_{k} T\right| & \geq k^{-\gamma} \min _{k<K(a)}\left|\frac{1-\cos \beta_{k} T}{k^{-\gamma}}\right| \\
& \geq C_{11} k^{-\gamma}>0, \quad k<K(a) .
\end{aligned}
$$

Thus, $\left|1-\cos \beta_{k} T\right| \geq C_{10} k^{-\gamma}$, where $C_{10}=\min \left\{2, C_{11}\right\}, \gamma>0$. Note that constant $C_{10}$ depends on $a$.

Theorem 5. For almost all (with respect to Lebesgue measure in $\mathbb{R}$ ) numbers $b \in\left[b_{1}, b_{2}\right]$ inequality

$$
\left|1-\cos \beta_{k} T\right| \geq C_{12} k^{-\gamma}
$$

is fulfilled for $\gamma>2$ and all $k \in \mathbb{N}$, where $C_{12}$ is a constant independent of $k$.

Theorem 6. For almost all (with respect to Lebesgue measure in $\mathbb{R}$ ) numbers $c \in\left[c_{1}, c_{2}\right]$ inequality

$$
\left|1-\cos \beta_{k} T\right| \geq C_{13} k^{-\gamma}
$$

is fulfilled for $\gamma>6$ and all $k \in \mathbb{N}$, where $C_{13}$ is a constant independent of $k$.

\subsection{The estimate in terms of parameter $T$.}

Lemma 4 ([12]). Let $\Phi(k)$ is bounded sequence of real numbers. Then for almost all (with respect to Lebesgue measure in $\mathbb{R}$ ) numbers $d>0$ inequality

$$
\left|\Phi(k)-\frac{m d}{|k|^{\sigma}}\right|<\frac{1}{|k|^{1+\sigma+\varepsilon}}
$$

where $0<\varepsilon<1, \sigma>0$, has no more than a finitely many integer solutions in $k \neq 0$ and $m \neq 0$.

Theorem 7. For almost all (with respect to Lebesgue measure in $\mathbb{R}$ ) numbers $T>0$ inequality

$$
\left|1-\cos \beta_{k} T\right| \geq 2 T^{2} k^{-\gamma}
$$

is fulfilled for $\gamma>2$ and all (except finitely many) numbers $k \in \mathbb{N}$.

Proof. Since $1-\cos \beta_{k} T=2 \sin ^{2} \beta_{k} \frac{T}{2}$ and $|\sin y| \geq \frac{2}{\pi}|y|$ for $|y| \leq \frac{\pi}{2}$, we can apply Lemma 4 . Then

$$
\left|1-\cos \beta_{k} T\right| \geq \frac{8}{\pi^{2}}\left|\beta_{k} \frac{T}{2}-\pi m\right|^{2}=2 T^{2} k^{4}\left|\frac{\beta_{k}}{k^{2} \pi}-\frac{2 m}{T k^{2}}\right|^{2} \geq \frac{2 T^{2} k^{4}}{|k|^{2(3+\varepsilon)}}=\frac{2 T^{2}}{k^{2+2 \varepsilon}}
$$

where $m \in \mathbb{N}$ such that $\left|\beta_{k} \frac{T}{2}-\pi m\right| \leq \frac{\pi}{2}, 0<\varepsilon<1, \gamma=2+2 \varepsilon>2$.

Thus, $\left|1-\cos \beta_{k} T\right| \geq 2 T^{2} k^{-\gamma}$, for $\gamma=2(\theta+\varepsilon)>2$ and all (except finitely many) numbers $k \in \mathbb{N}$. 
Theorem 8. For almost all (with respect to Lebesgue measure in $\mathbb{R}$ ) numbers $T>0$ condition (9) is fulfilled.

Corollary 2. For almost all (with respect to Lebesgue measure in $\mathbb{R}$ ) numbers $T>0$ inequality

$$
\left|1-\cos \beta_{k} T\right| \geq C_{14} k^{-\gamma}
$$

is fulfilled for $\gamma>2$ and all numbers $k \in \mathbb{N}$, where $C_{14}$ is a constant independent of $k$.

Corollary 3. If $\varphi \in \mathbf{H}_{q+\rho+2}$ and $\psi \in \mathbf{H}_{q+\rho}$, where $\rho>2$, then for almost all (with respect to Lebesgue measure in $\mathbb{R}$ ) numbers $a \in\left[a_{1}, a_{2}\right]$ there exists a unique solution $u \in \mathbf{C}^{2}\left([0, T] ; \mathbf{H}_{q}\right)$ of the problem (1)-(3).

\section{REFERENCES}

[1] Azizbayov E., Mehraliyev Y. A boundary value problem for equation of homogeneous bar with periodic conditions. Amer. J. Appl. Math. Stat. 2015, 3 (6), 252-256. doi: 10.12691/ajams-3-6-6

[2] Azizbayov E., Mehraliyev Y. A time-nonlocal boundary value problem for equation of homogeneous bar motion. Bull. Kyiv National Univ. Ser. Math. Mekh. 2012, 27, 20-23.

[3] Bors I., Milchis T. Dynamic response of beams on elastic foundation with axial load. Acta Tech. Napocensis: Civil Eng. \& Arch. 2013, 56 (1), 67-81.

[4] Chandra T.K. The Borel-Cantelli Lemma. In: Ahmad Sh. SpringerBriefs in Statistics, 2. Springer, India, 2012.

[5] Goi T.P., Ptashnik B.I. Nonlocal boundary-value problems for systems of linear partial differential equations with variable coefficients. Ukrainian Math. J. 1997, 49 (11), 1659-1670. doi: 10.1007/BF02487504 (translation of Ukrain. Mat. Zh. 1997, 49 (11), 1478-1487 (in Ukrainian)).

[6] Goi T.P., Ptashnik B.I. Problem with nonlocal conditions for weakly nonlinear hyperbolic equations. Ukrainian Math. J. 1997, 49 (2), 204-215. doi: 10.1007/BF02486436 (translation of Ukrain. Mat. Zh. 1997, 49 (2), 186-195. (in Ukrainian))

[7] Il'kiv V.S., Nytrebych Z.M., Pukach Ya. Boundary-value problems with integral conditions for a system of Lame equations in the space of almost periodic functions. Electron. J. Differential Equations 2016, 2016 (304), 1-12.

[8] Il'kiv V.S., Ptashnyk B.I. Problems for partial differential equations with nonlocal conditions. Metric approach to the problem of small denominators. Ukrainian Math. J. 2006, 58 (12), 1847-1875. doi: 10.1007/s11253-006-0172-8 (translation of Ukrain. Mat. Zh. 2006, 58 (12), 1624-1650. (in Ukrainian))

[9] Kalenyuk P.I., Kohut I.V., Nytrebych Z.M. Problem with nonlocal two-point condition in time for a homogeneous partial differential equation of infinite order with respect to space variables. J. Math. Sci. 2010, 167 (1), 1-15. doi: 10.1007/s10958-010-9898-9 (translation of Mat. Metody Fiz.-Mekh. Polya 2008, 51 (4), 17-26. (in Ukrainian))

[10] Kostin D.V. Application of Maslov's formula to finding an asymptotic solution to an elastic deformation problem. Math. Notes 2008, 83 (1-2), 48-56. doi: 10.1134/S0001434608010069 (translation of Mat. Zametki 2008, 83 (1), 50-60. (in Russian))

[11] Nakhushev A.M. Nonlocal boundary problems with displacement and their relation to loaded equations. Differ. Equations 1985, 21 (1), 92-101. (in Russian)

[12] Ptashnyk B.Yo., Il'kiv V.S., Kmit' I.Ya., Polishchuk V.M. Nonlocal boundary value problems for partial differential equations. Naukova Dumka, Kyiv, 2002. (in Ukrainian)

[13] Sabitov K.B. Fluctuations of a beam with clamped ends. J. Samara State Tech. Univ. Ser. Phys. Math. Sci. 2015,19 (2), 311-324. doi: 10.14498/vsgtu1406 (in Russian)

[14] Savka I.Y. A nonlocal boundary-value problem for partial differential equations with constant coefficients belonging to smooth curves. J. Math. Sci. 2011, 174 (2), 136-158. doi: 10.1007/s10958-011-0286-x (translated of Mat. Metody Fiz.-Mekh. Polya 2009, 52 (4), 18-33. (in Ukrainian)) 
[15] Symotyuk M.M., Savka I.Ya. Initial-nonlocal boundary value problems for factorized partial differential equations. Bull. Lviv Polytech. National Univ. Ser Phys. \& Math. Sci. 2013, 768, 19-25. (in Ukrainian)

[16] Symotyuk M.M., Savka I.Y. Metric estimates of small denominators in nonlocal boundary value problems. Bull. South Ural State Univ. Ser. Math. Mechanics. Phys. 2015, 7 (3), 48-53. (in Russian)

[17] Vasylyshyn P.B., Savka I.Ya., Klyus I.S. Multipoint nonlocal problem for factorized equation with dependent coefficients in conditions. Carpathian Math. Publ. 2015, 7 (1), 22-27. doi: 10.15330/cmp.7.1.22-27

[18] Vlasii O.D., Goy T.P., Ptashnyk B.Yo. A problem with nonlocal conditions for weakly nonlinear equations with variable coefficients in the principal part of the operator. Mat. Metody Fiz.-Mekh. Polya 2004, 47 (4), 101-109. (in Ukrainian)

[19] Vlasii O.D., Goy T.P., Savka I.Ya. Boundary value problem with nonlocal conditions of a second kind for the hyperbolic factorized operator. Uzhhorod Univ. Sci. Bull. Ser. Math. \& Inform. 2014, 25 (1), 33-46. (in Ukrainian)

Received 31.10.2017

Revised 11.06.2018

Гой Т., Негрич М., Савка І. Про нелокальну крайову задачу для рівняння руху однорідної еластииної балки із нежорстко закріпленими кіниями // Карпатські матем. публ. - 2018. - Т.10, №1. - С. 105-113.

В області $D=\{(t, x): t \in(0, T), x \in(0, L)\}$ досліджено крайову задачу для рівняння руху однорідної еластичної балки

$$
u_{t t}(t, x)+a^{2} u_{x x x x}(t, x)+b u_{x x}(t, x)+c u(t, x)=0,
$$

де $a, b, c \in \mathbb{R}, b^{2}<4 a^{2} c$, з нелокальними двоточковими умовами

$$
u(0, x)-u(T, x)=\varphi(x), \quad u_{t}(0, x)-u_{t}(T, x)=\psi(x)
$$

і локальними крайовими умовами $u(t, 0)=u(t, L)=u_{x x}(t, 0)=u_{x x}(t, L)=0$. Розв'язність цієї задачі пов'язана з проблемою малих знаменників, для оцінки знизу яких застосовується ме-

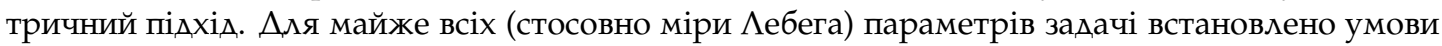
розв'язності задачі в просторах Соболєва. Зокрема, якшо $\varphi \in \mathbf{H}_{q+\rho+2}$ i $\psi \in \mathbf{H}_{q+\rho}$, де $\rho>2$,

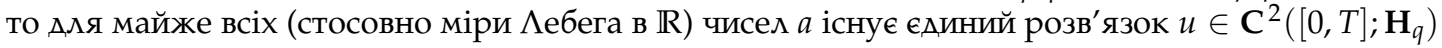
задачі.

Ключові слова і фрази: нелокальна крайова задача, однорідна балка, малий знаменник, міра Аебега, метричний підхід. 